\title{
Capacidade preditiva dos componentes do lucro e incerteza política: evidências de países da América Latina
}

\author{
Lineker Costa Passos \\ https://orcid.org/0000-0002-1317-9631 \\ Paulo Roberto Nóbrega Cavalcante \\ https://orcid.org/0000.0002.1702.0433
}

\section{Resumo}

Objetivo: Examinar se a capacidade dos componentes fluxo de caixa e accruals atuais em predizer futuros fluxos de caixa é afetada em períodos de incerteza política.

Método: Considerou-se o ano de eleições nacionais como proxy para incerteza política, ao passo que a equação de previsão de fluxos de caixa de um período à frente, estimada por Sys-GMM, foi utilizada para captar a capacidade preditiva dos componentes do lucro segundo períodos de incerteza política.

Amostra: Constituída por 386 firmas (4.127 observações-ano) listadas nas bolsas da Argentina, do Brasil, do Chile e do México.

Resultados: A capacidade preditiva do componente fluxo de caixa atual sofreu influência negativa de períodos de incerteza política, ao passo que a capacidade do componente accruals não se mostrou afetada por aqueles períodos. Conclui-se que incerteza política afeta negativamente a capacidade preditiva dos componentes do lucro divulgado, contudo tal efeito se mostrou conclusivo apenas para o componente fluxo de caixa atual.

Contribuições: Apresentação da realidade do mercado de capitais de países emergentes se constitui na principal contribuição do estudo. Ademais, adiciona-se conhecimento à literatura relacionada à hipótese de incerteza política, lançando luz sobre o impacto desse evento na oferta e disponibilidade de informações úteis em mercado de capitais de países emergentes. Também se mostra relevante para agentes que realizam previsões sobre fluxos de caixa futuros das firmas.

Palavras-chave: Incerteza política; Capacidade preditiva dos lucros; Accruals; Fluxos de caixa. 


\section{Introdução}

Diversas pesquisas (Ashton \& Trinh, 2018; Ball \& Brown, 1968; Beaver, 1968; Beaver, Mcnichols \& Wang, 2018) têm confirmado a relevância da informação contábil para agentes atuantes no mercado de capitais, tendo como base o direcionador das decisões dos agentes que atuam naquele mercado, qual seja, os fluxos futuros de caixas das organizações.

Um dos principais argumentos para tal relevância refere-se ao valor preditivo da informação contábil, segundo Wolk, Dodd e Tearney (2004). Nessa direção, Barth, Cram e Nelson (2001) argumentam que a capacidade de uma firma gerar fluxos de caixas afeta o valor de seus títulos e que, por esta razão, o Financial Accounting Standards Board (Fasb) indica que o objetivo primário da contabilidade é prover informação para ajudar investidores, credores e outros usuários a avaliarem fluxos de caixas futuros.

Nesse contexto, evidências empíricas (Dechow, Kothari \& Watts, 1998; Jordan, Waldron \& Clark, 2007; Kim \& Kross, 2005) têm confirmado os lucros divulgados pelos sistemas de contabilidade das firmas - tidos como sinal informacional ótimo para direcionar as decisões de alocação de recursos no mercado de capitais (Lev, 1989) - como efetivos preditores de fluxos de caixa futuros.

Outros estudos (Barth, Cram \& Nelson, 2001; Boina \& Macedo, 2018; Lev, Li \& Sougiannis, 2010), de outra parte, têm sinalizado aumento da força preditiva de modelos de previsão de futuros fluxos de caixa ao se considerar nestes os componentes segregados dos lucros divulgados, quais sejam, os componentes accruals e fluxo de caixa. Ressaltem-se, ainda, evidências sinalizando a maior capacidade preditiva do componente fluxo de caixa em comparação ao componente accruals, conforme sinalizado por Sloan (1996).

Um dos fatores que podem ser usados para explicar essa assimetria de força preditiva entre os componentes fluxo de caixa atual e accruals é a discricionariedade de gestores quanto ao reconhecimento de accruals. Barth, Beaver, Hand e Landsman (1999), a esse respeito, argumentam que o componente accruals carrega maior grau de subjetividade que o componente fluxo de caixa, pelo fato de poder ser objeto da discricionariedade de gestores; o reconhecimento de accruals discricionários é provável de conter itens não usuais, provável a não se repetir no futuro, o que impacta diretamente a capacidade dos accruals em predizer futuros fluxos de caixa. Apesar de menos suscetível que os accruals, o componente fluxo de caixa também pode ser alvo da discricionariedade de gestores no intuito de gerar fluxos de caixa atuais, insustentáveis no futuro, para se alcançar determinado alvo (Roychowdhury, 2006), o que também impacta a capacidade desse componente em predizer futuros fluxos de caixa.

Na ocorrência dessas situações, lucros divulgados se desviam de sua função precípua de refletir a realidade econômica subjacente esperada, caracterizando-se o que se conhece na literatura por lucros de baixa qualidade informacional (Dechow, Ge \& Schrand, 2010). Pode-se afirmar, portanto, que a capacidade dos lucros atuais em predizer futuros fluxos de caixa também é dependente da qualidade informacional de seus componentes.

Destaca-se que o nível de discricionariedade dos gestores pode refletir o efeito de eventos outros que não aqueles diretamente vinculados ao negócio, afetando mais acentuadamente a qualidade dos componentes do lucro e, portanto, a capacidade preditiva tanto do fluxo de caixa, quanto dos accruals atuais, isto se tais eventos incrementam a incerteza sobre a performance futura das firmas. Nesse contexto, Leal, Girão, Lucena e Martins (2017) apresentaram evidências, no contexto do mercado de capitais brasileiro, de que valores extremos de lucros afetam a capacidade preditiva dos componentes dos lucros divulgados. Explicam os autores que tanto lucros quanto fluxos de caixa, em situações extremas, são menos persistentes e, em decorrência disso, apresentam maior volatilidade; concluem que, em situações extremas, tanto lucros (e seus componentes) quanto fluxos de caixa apresentam menor capacidade preditiva. 
Outro possível atributo com capacidade de incrementar a incerteza associada à performance futura das firmas refere-se a períodos de incerteza política (Brogaard \& Detzel, 2015). Incerteza política é definida por Chen, Hope, Li e Wang (2018) como prováveis mudanças de governo e de políticas de governo, e tem sido caracterizada por sua capacidade de incrementar a incerteza sobre futuros fluxos de caixa (Dai \& Ngo, 2020), uma vez que pode incrementar sua volatilidade (Boutchkova, Doshi, Durnev \& Molchanov, 2012; Brogaard \& Detzel, 2015). Ressalte-se que a maior volatilidade do fluxo de caixa reduz sua capacidade de predizer a performance futura da firma (Minton, Schrand \& Walther, 2002).

Nesse cenário, a incerteza política poderia estar afetando negativamente a capacidade preditiva do componente fluxo de caixa, dado que incrementaria a volatilidade desse componente. Tal efeito também pode ser esperado para o componente accruals, uma vez que a volatilidade dos fluxos de caixa também contribui para accruals de menor capacidade preditiva (Dechow \& Dichev, 2002). Mais ainda, dada a maior volatilidade em períodos de incerteza política, maior a possibilidade de erros quando da estimação de accruals, o que reduz a capacidade desse componente dos lucros divulgados em predizer futuros fluxos de caixa, conforme Dechow e Dichev (2002).

Ademais, Yung e Root (2019), examinando o efeito da incerteza política na prática de gerenciamento de resultados em amostra de 18 países, apontaram evidências de que gestores optam por escolhas contábeis no intuito de manipular tanto o componente accruals quanto o componente fluxo de caixa seguindo períodos de maior incerteza política, o que também reduz a força preditiva de ambos os componentes do lucro divulgado.

Nesses termos, tanto o incremento de volatilidade no componente fluxo de caixa quanto a maior possibilidade de manipulação dos componentes do lucro, associados ao evento incerteza política, podem reduzir a capacidade dos componentes fluxo de caixa e accruals em predizer futuros fluxos de caixa, uma vez que é provável que ambos os componentes atuais afetados pela incerteza política não se repitam no futuro período.

Não se localizaram, embora em pesquisa não exaustiva, estudos voltados para investigação de tal questão. Chen, Chen, Wang e Zheng (2018), a esse respeito, destacam baixo volume de evidências empíricas sobre os impactos da incerteza política no fornecimento e disponibilidade de informações relevantes a investidores nos mercados de capitais; os autores ressaltam a importância de tal questão, dado que a disponibilidade de informações relevantes é fator-chave para a eficiente alocação de recursos e essencial para as decisões de investimentos de investidores.

O exame de tal questão se torna relevante no contexto de mercados emergentes, uma vez que a incerteza política apresenta maior efeito negativo em mercados de capitais emergentes do que em mercados de capitais desenvolvidos, conforme Diamonte, Liew e Steven (1998). Ressalte-se que o ambiente institucional de países da América Latina é geralmente caracterizado por fracos mecanismos de enforcement e de proteção a investidores (Brown, Preiato \& Tarca, 2014; La Porta, Lopez-de-Silanes, Shleifer, \& Vishny, 1998; Moura, Altuwaijri \& Gupta, 2020). Em tal configuração, gestores podem ter mais incentivos para reportar discricionariamente os componentes do lucro em períodos de incerteza política, o que se refletiria em menor capacidade preditiva dos componentes do lucro divulgado.

Sob tal contexto, busca-se coletar evidências que respondam à seguinte questão: em que medida a incerteza política afeta a capacidade dos componentes do lucro divulgado - accruals e fluxos de caixa - em predizer futuros fluxos de caixa?

A investigação se mostra relevante por captar efeito pouco documentado no contexto internacional, qual seja, a influência da incerteza política na capacidade preditiva dos componentes do lucro divulgado no âmbito de mercados emergentes, preenchendo-se, portanto, relevante lacuna de pesquisa. Esta inova por considerar o objeto de pesquisa em países específicos da América Latina, diferenciando-se, portanto, da pesquisa de Yung e Root (2019). Além disso, a pesquisa também se diferencia por considerar os efeitos adversos da incerteza política em medida que também reflete a qualidade dos agregados contábeis não considerada naquele estudo, qual seja, a capacidade preditiva dos componentes do lucro divulgado. 
A pesquisa contribui para incremento da literatura relacionada à hipótese de incerteza política, conforme já relatada em outras investigações (Brogaard \& Detzel, 2015; Dai \& Ngo, 2020). Destaque-se, ainda, que a pesquisa também fornece contribuições à literatura relacionada à qualidade da informação contábil, especificamente por apresentar evidências que complementam Chen, Chen et al. (2018), Leal et al. (2017) e Yung e Root (2019) sobre o impacto negativo de evento exógeno às firmas na qualidade das informações contábeis divulgadas por gestores.

Ademais, o estudo também se mostra relevante para investidores, analistas e outros agentes de mercado que realizam previsões sobre os fluxos de caixa futuros das firmas, no sentido de lhes prover evidências acerca da relevância de se considerar os efeitos advindos da incerteza política no contexto daquelas previsões, uma vez que as evidências apontaram para impacto adverso significante da incerteza política na capacidade do componente fluxo de caixa atual em predizer futuros fluxos de caixa.

\section{Referencial Teórico e Desenvolvimento da Hipótese}

Desde os trabalhos seminais de Ball e Brown (1968) e Beaver (1968), pesquisas (Ashton \& Trinh, 2018; Beaver et al., 2018) têm apresentado evidências de que a informação contábil é relevante para os agentes dos mercados de capitais. O principal argumento para essa relevância reside no valor preditivo da informação contábil ou na sua capacidade de predizer fluxos de caixa futuros (Wolk et al., 2004).

Alinhados ao argumento do valor preditivo da informação contábil, pesquisadores (Barth et al., 2001; Boina \& Macedo, 2018; Lev et al., 2010) têm apresentado evidências de que os componentes do lucro divulgado - fluxos de caixa e accruals do período atual - apresentam capacidade marginal em predizer fluxos de caixa futuros. As evidências apresentadas sinalizaram incremento da força preditiva em modelos de previsão de futuros fluxos de caixa quando da inclusão tanto do componente fluxo de caixa quanto do componente accruals.

Apesar do incremento de força preditiva, vale ressaltar que tanto o componente fluxo de caixa quanto o componente accruals podem ser objetos da discricionariedade de gestores, com maior grau de possibilidade para o componente accruals (Barth et al., 1999). Essa discricionariedade por parte de gestores pode fazer com que os componentes do lucro divulgado contenham itens não usuais que não se repetirão no futuro, o que impactará a capacidade desses componentes de refletir a performance econômica subjacente esperada. Sob essa hipótese, lucros são caracterizados como de baixa qualidade informacional (Dechow et al., 2010) e falham ao desempenhar sua função em predizer eficientemente futuros fluxos de caixa.

Adicionalmente ao efeito oriundo da discricionariedade dos gestores, outros atributos com capacidade para afetar a capacidade preditiva de ambos os componentes do lucro divulgado, fluxos de caixa e accruals, se referem a eventos que incrementam a incerteza associada à performance futura das firmas.

Leal et al. (2017), a esse respeito, examinaram a capacidade dos componentes do lucro divulgado em predizer futuros lucros em situações de lucros extremos no mercado de capitais brasileiro. Lucros extremos, segundo os autores, foram mensurados dividindo-se a variável lucro em decis, sendo considerados extremos os decis 1,2 e 9 . As evidências sinalizaram que a capacidade preditiva do componente fluxo de caixa nos decis 1 e 2 é menor do que a força preditiva do componente accruals; tal situação se inverte no decil 9, em que a força preditiva do componente fluxo de caixa supera o valor preditivo de accruals. Os achados sugerem que, em situações de valores extremos negativos (decil 1), accruals são mais preditivos que fluxos de caixa; já em situações de valores extremos positivos (decil 9), accruals são menos preditivos que fluxos de caixa.

Concluem os autores que situações de lucros e fluxos de caixa extremos afetam o valor preditivo tanto dos lucros quanto do fluxo de caixa, com efeito negativo mais pronunciado em fluxos de caixa. Mais ainda, apresentam evidências de que accruals é o componente que reduz a capacidade preditiva dos lucros. 
Ocorre que pesquisas sobre impacto de situações de performance extrema na capacidade preditiva dos componentes do lucro divulgado podem sofrer de questões relacionadas à endogeneidade, uma vez que tal performance pode ser determinada endogenamente pelo uso dos componentes do lucro; por exemplo, gestores podem utilizar a discricionariedade de accruals para gerar performances extremas, na hipótese de haver incentivos para tal prática.

Outro provável evento com capacidade de influenciar marginalmente a performance futura da firma e menos provável de ser determinado por atributos individuais das firmas, mitigando potenciais problemas de endogeneidade, refere-se à incerteza política.

Tal atributo tem sido apontado como relevante por impactar de maneira adversa a atividade econômica e os resultados financeiros. A esse respeito, pesquisas têm sinalizado efeitos adversos da incerteza política nos preços de ativos (Gao \& Qui, 2014; Pastor \& Veronesi, 2012) e nas decisões corporativas (An, Chen, Luo \& Zhang, 2016; Julio \& Yook, 2012), o que tem sido caracterizado como Hipótese da Incerteza Política (Julio \& Yook, 2012). É provável que, dada a possibilidade de choques adversos no valor futuro do ativo - fluxos futuros de caixa, agentes do mercado descontariam o preço atual para refletir tal choque adverso.

Dai e Ngo (2020) afirmam que possíveis mudanças de liderança política e/ou políticas de governo incrementam a incerteza sobre fluxos de caixa futuros da firma; evidências fornecidas por Boutchkova et al. (2012) e Brogaard e Detzel (2015) confirmam tal argumento, sinalizando a maior volatilidade de fluxos de caixa futuros em períodos de incerteza política. Ocorre que maior volatilidade se associa negativamente a performance futura da firma, conforme Minton et al. (2002); os autores evidenciaram que a maior volatilidade do fluxo de caixa operacional reduz sua capacidade de predizer a performance futura. Ademais, maior volatilidade implica menor persistência do componente fluxo de caixa e, portanto, menor capacidade preditiva desse componente em predizer fluxos de caixa futuros.

Nesse sentido, a incerteza política poderia estar afetando negativamente a capacidade preditiva do componente fluxo de caixa, dado que incrementaria a volatilidade desse componente e, portanto, reduziria sua persistência temporal.

Tal efeito também pode ser esperado para o componente accruals. Ocorre que, na hipótese de maior incerteza política, é provável que erros (intencionais ou não) ocorram com maior frequência na estimativa de accruals, uma vez que aquela incerteza introduz volatilidade no ambiente das firmas, e que tal volatilidade afeta a qualidade de estimação de accruals e, portanto, a capacidade preditiva de accruals, conforme Dechow e Dichev (2002).

Dentre os atributos que também contribuiriam para accruals de menor capacidade preditiva, Dechow e Dichev (2002) destacam a volatilidade dos fluxos de caixa; argumentam que a maior volatilidade desse componente do lucro reflete medida de alta incerteza, e que tal atributo contribui para accruals com menor capacidade para predizer fluxos de caixa futuros.

Ademais, é provável que o componente accruals seja mais suscetível à discricionariedade de gestores que o componente fluxo de caixa (Barth et al., 1999); gestores podem estimar accruals discricionários contendo itens não usuais que provavelmente não se repetirão no futuro, o que reduz sua capacidade em predizer futuros fluxos de caixa.

Nessa direção, Yung e Root (2019) apresentaram evidências de maior uso de accruals discricionários em períodos de incerteza política em amostra de 18 países no período 2001-2014. Accruals discricionários foram estimados segundo os modelos Jones (1991), Dechow e Dichev (2002), Dechow, Sloan e Sweeney (1995) e Dechow e Dichev (2002), modificados por McNichols (2002), ao passo que a incerteza política foi mensurada considerando-se o índice de incerteza política de Baker, Bloom e Davis (2016). As evidências apontaram para forte uso de accruals discricionários quando a incerteza política é alta. 
Ressalte-se que o componente fluxo de caixa também pode ser alvo da discricionariedade de gestores (Roychowdhury, 2006); gestores podem gerar fluxos de caixa insustentáveis para se alcançar determinado alvo (Roychowdhury, 2006), o que afeta negativamente sua capacidade em predizer futuros fluxos de caixa. Yung e Root (2019), em testes adicionais, também apresentaram evidências de manipulação dos fluxos de caixa seguindo períodos de maior incerteza política.

Baseando-se nas evidências de Yung e Root (2019), espera-se que, na hipótese de escolhas discricionárias por parte de gestores quanto aos componentes fluxo de caixa e accruals diante de maior incerteza política, os referidos componentes exibam menor capacidade em predizer futuros fluxos de caixa em períodos de incerteza política.

Ainda no contexto de incremento de discricionariedade de gestores em períodos de incerteza política, convém destacar que o ambiente institucional de países da América Latina pode fornecer incentivos na direção daquele incremento. A literatura tem caracterizado esse ambiente exibindo fracos mecanismos de enforcement e de proteção a investidores (Brown et a., 2014; La Porta et al., 1998; Moura et al., 2020), e que tais características se associam a práticas oportunistas executadas por gestores (Leuz, Nanda \& Wysocki, 2003). Nesse contexto, se poderia esperar maiores incentivos às práticas discricionárias de gestores em períodos de incerteza política, o que poderia refletir em impacto na capacidade preditiva dos componentes do lucro.

Vale ressaltar que não há consenso na literatura quanto à proxy direta e eficiente para o atributo incerteza política. Julio e Yook (2012) sinalizam o evento eleições nacionais como atributo que disponibiliza configuração interessante para pesquisadores. Conforme os autores, eleições nacionais são relevantes para decisões corporativas, dado que podem afetar regulação das indústrias, políticas monetárias e de comercialização, tributação e, em casos mais extremos, possível expropriação ou nacionalização de firmas privadas.

Chen, Hope et al. (2018), por sua vez, argumentam que eleições nacionais refletem alta incerteza política porque podem romper o equilíbrio econômico e político estabelecido, bem como afetar significativamente a decisão de alocação de recursos. Ademais, eleições nacionais são definidas de forma exógena a qualquer firma individual (Julio \& Yook, 2012), o que pode aliviar potenciais problemas de endogeneidade na pesquisa.

Por todo o exposto, a incerteza política pode impactar a capacidade preditiva dos componentes dos lucros divulgados segundo os seguintes argumentos: (i) incremento de volatilidade do componente fluxo de caixa em períodos de maior incerteza política, que pode impactar tanto a capacidade do componente fluxo de caixa atual quanto a do componente accruals em predizer futuros fluxos de caixa; (ii) escolhas discricionárias de gestores no sentido de manipular os componentes fluxo de caixa atual e accruals em períodos de incremento de incerteza política, o que reduz a capacidade preditiva de ambos os componentes do lucro divulgado. Considerando-se essa argumentação, adotou-se a seguinte hipótese:

H1: A incerteza política afeta negativamente a capacidade dos componentes do lucro divulgado - accruals e fluxo de caixa atuais - em predizer futuros fluxos de caixa.

\section{Procedimentos Metodológicos}

A população da pesquisa congregou firmas listadas nas bolsas de valores de seis países emergentes da América Latina, quais sejam: Argentina, Brasil, Chile, Colômbia, México e Peru (Buenos Aires Stock Exchange, Brasil, Bolsa, Balcão - B3, Santiago Stock Exchange, Colômbia Stock Exchange, Bolsa de Valores de Lima e Bolsa Mexicana de Valores, respectivamente), tomando por base classificação proposta pelo Fundo Monetário Internacional [FMI] (2018). Moreira (2018) aponta a relevância econômica destes países no contexto latino-americano, destacando-os como representantes dos maiores Produtos Internos Brutos (PIBs) em 2016, segundo o Banco Mundial (2018). 
A amostra, por seu turno, constituiu-se de firmas listadas na Argentina, no Brasil, no Chile e no México, após exclusão de firmas listadas na Colômbia, por haver menos de 100 empresas listadas em seu mercado de capitais (World Federation of Exchanges, 2018), bem como por haver grande quantidade de dados faltantes para as firmas, conforme os critérios de Paulo, Martins e Girão (2014). Também foram excluídas firmas listadas no Peru, uma vez que se constatou baixa representatividade desse mercado em relação aos demais da amostra; para ilustrar esse argumento, com base em dados do World Federation of Exchanges (2018), constatou-se que, até o ano de 2006, o mercado de capitais peruano exibiu capitalização média de apenas $42 \%$ da capitalização do mercado argentino, sendo este o mercado de menor capitalização dentre os países da amostra, o que pode se refletir em incentivos muito distintos entre mercados, uma vez que, em mercados menores, fatores não econômicos podem ser mais relevantes que fatores econômicos (Alexakis \& Petrakis, 1991). Ademais, também foram excluídas observações por ausência de dados e por pertencerem às firmas do setor financeiro, visto que suas práticas de divulgação diferem da prática usual de firmas dos demais setores (Pincus, Rajgopal \& Venkatachalam, 2007). A amostra final ficou constituída por 386 firmas (4.127 observações-ano), conforme se detalha na Tabela 1:

Tabela 1

Formação da amostra por países

\begin{tabular}{cccccc}
\hline & Amostra total & Argentina & Brasil & Chile & \multicolumn{1}{c}{ México } \\
\hline Firmas & 386 & 43 & 221 & 34 & 88 \\
\hline Observações-ano & 4.127 & 469 & 2.507 & 408 & 743 \\
\hline
\end{tabular}

Fonte: dados da pesquisa.

A investigação compreendeu eventos observados durante o período de 1998 a 2018. Tal escolha levou em consideração a captação de possíveis efeitos de diversos períodos eleitorais nas firmas dos países da amostra.

Os dados necessários para o processamento da pesquisa foram coletados: a) na base Thomson Reuters ${ }^{\circledR}$, esta utilizada para coleta de informações contábeis; b) nas bases Data e Database of Political Institutions presentes no The World Bank, tais bases utilizadas para coleta relacionadas às variações no PIB dos países e aos períodos eleitorais nacionais, respetivamente; c) na base da plataforma IFRS (ifrs.org), esta utilizada para captar os períodos de convergência ao padrão IFRS de cada país da amostra.

Processou-se winsorização dos dados, considerando-se os percentis 5 e 95, seguindo Monfared e Pavlov (2019), de forma a se mitigar efeitos de outliers. Ressalte-se que foram realizados testes adicionais considerando-se winsorização dos dados nos percentis 1 e 99, bem como também considerando-se os dados sem qualquer processo de winsorização. Contudo, as estimativas se mostraram sensíveis a essas escolhas e não exibiram estimativas consistentes para os parâmetros e testes. Tal constatação possivelmente explicada pela elevada dispersão das principais variáveis do estudo e pela presença de outliers nestas variáveis; ressalte-se que esses problemas apenas se apresentaram mais fortemente mitigados ao se considerar percentis 5 e 95 no processo de winsorização.

A incerteza política foi mensurada considerando períodos de eleições nacionais, seguindo Chen, Hope et al. (2018) e Julio e Yook (2012). Para tanto, utilizou-se variável dicotômica (ELE) para designar a presença de períodos de eleições nacionais, a qual assume 1 para o ano de ocorrência das eleições nacionais ou 0, caso contrário, seguindo Julio e Yook (2012). 
Para examinar a hipótese de que a incerteza política afeta negativamente a capacidade dos componentes do lucro divulgado em predizer futuros fluxos de caixa, utilizou-se a equação de previsão de fluxos de caixas operacionais de um período à frente (Barth, Clinch \& Israeli, 2016; Hope, Thomas \& Vyas, 2016), conforme modelo abaixo:

$$
\begin{gathered}
F C_{i, t+1, k}=\beta_{0}+\beta_{1} F C_{i, t, k}+\beta_{2} A C C_{i, t, k}+\beta_{3} E L E_{t, k}+\beta_{4} F C_{i, t, k} * E L E_{t, k}+ \\
\beta_{5} A C C_{i, t, k} * E L E_{t, k}+\sum_{n=6}^{N} \beta_{n} \text { CONTROLES }+\varepsilon_{i t}
\end{gathered}
$$

Em que $F C_{i, t+1, k}$ é o fluxo de caixa operacional do período $t+1$ para a firma $i$ e no país $k$, escalado pelo ativo total de $t$-1 da firma $i ; F C_{i, t, k}$ é o fluxo de caixa operacional do período $t$ para a firma $i$ e no país $k$, escalado pelo ativo total de $t-1$ da firma $i ; A C C_{i, t, k}$ são os accruals no período $t$ para a firma $i$ e no país $k$; $E L E_{t, k}$ é variável dummy representativa de ano de eleições nacionais no país $k$, assumindo 1 para esses anos, e 0 em caso contrário; $E L E_{t, k} * F C_{i, t, k}$ representa interação para $F C_{i, t, k}$ em anos de eleições nacionais; $E L E_{t, k} * A C C_{i, t, k}$ representa interação para $A C C_{i, t, k}$ em anos de eleições nacionais; CONTROLES se refere à matriz de variáveis de controles utilizadas, quais sejam, tamanho (TAM) e crescimento (CRESC) da firma, dummy representativa do período IFRS, a variação anual do Produto Interno Bruto (PIB) e dummies para países, anos e setores considerados na amostra; $\varepsilon_{t}$ é o termo de erro que capta os resíduos da regressão.

Accruals do período foram mensurados utilizando-se como proxy a variação da Necessidade de Capital de Giro (NCG), segundo Dechow, Hutton, Kim e Sloan (2012), conforme se expressa na equação conforme a equação (2):

$$
A C C_{t}=\left[\left(\Delta A C_{t}-\Delta A F_{t}\right)-\left(\Delta P C_{t}-\Delta P F_{t}\right)\right] / A T_{t-1}
$$

Em que $A C C t$ são os accruals no período escalados pelo ativo total de t-1; $(\triangle A C-\triangle A F)$ representa o incremento dos Ativos Circulantes Operacionais no período; $(\triangle P C-\triangle P F)$ representa o incremento nos Passivos Circulantes Operacionais no período; $A T_{t-1}$ corresponde ao Ativo Total no período $t-1$.

O fluxo de caixa operacional, por sua vez, foi mensurado pela diferença entre o Lucro Antes de Juros e Impostos sobre o Lucro (LAJIR), escalado pelo ativo total de $t$-1, e accruals do período estimados conforme a equação (2), seguindo Passos e Coelho (2019).

Ressalte-se que o cálculo indireto das variáveis accruals e fluxo de caixa decorre da inexistência de Demonstração de Fluxo de Caixa em todo o período abordado, o que impede a utilização da abordagem de fluxo de caixa para obtenção direta das medidas.

O tamanho da firma (TAM) foi mensurado pelo log natural do ativo total da firma, ao passo que a medida crescimento da firma (CRESC) foi mensurada pela variação percentual da receita líquida entre os períodos $t$ e $t$ - 1 . Ambas medidas representam controle para efeitos advindos de características das firmas, conforme já reportado em pesquisas anteriores (Choi, Han, Jung \& Kang, 2015; Farshadfar, Ng \& Brimble, 2008). Espera-se que TAM e CRESC se associem positivamente e negativamente a futuros fluxos de caixa, respectivamente, uma vez que firmas maiores e com menor crescimento exibem características operacionais mais estáveis e que contribuem para sua maior capacidade preditiva de futuros fluxos de caixa quando comparadas a firmas menores e com alto crescimento.

Incluiu-se variável binária representativa do período após convergência ao padrão IFRS no modelo (1), considerando-se os diferentes períodos de convergência de cada país da amostra, no intuito de se controlar possíveis efeitos advindos da mudança de padrão contábil, conforme já reportado em Machado, Silva Filho e Callado (2014) e Boina e Macedo (2018); considerou-se, com suporte na plataforma IFRS (ifrs.org), os seguintes períodos de convergência: Argentina - início em 2012, Brasil - início em 2010, Chile - início em 2009 e México - início em 2012. 
Também foram incluídas dummies para se controlar possíveis efeitos temporais, dos diferentes setores das firmas, dos diferentes países da amostra, bem como variável representativa da variação do PIB nacional para se controlar possíveis efeitos de choques econômicos.

Espera-se que os coeficientes $\beta_{4}$ e $\beta_{5}$ da regressão (1) sejam negativos e significantes, na hipótese de impacto negativo da maior incerteza política na capacidade dos componentes do lucro em predizer futuros fluxos de caixa.

O modelo foi processado por meio de regressão linear múltipla com estimação pelo System Generalized Method of Moments (Sys-GMM), o qual se baseia na estimação por meio de modelos dinâmicos (Barros, Castro, Silveira \& Bergmann, 2020); tal método se apresenta mais consistente em relação a outros métodos quando da existência de problemas de endogeneidade e de autocorrelação serial (Barros et al., 2020).

A regressão (1) foi processada com correção de variância para amostras finitas, de forma a se corrigir possível heterocedasticidade dos resíduos, conforme Windmeijer (2005). Examinou-se existência de multicolinearidade entre as variáveis independentes com análise de correlação, bem como problemas de autocorrelação dos resíduos por meio dos testes de Arellano e Bond (1991); ambos os problemas ausentes, segundo os testes realizados (não reportados). Aplicou-se o Hansen Test, o qual não se mostrou significante, sinalizando que os instrumentos utilizados na estimação do modelo (1) são válidos, confirmando-se a consistência de estimação por Sys-GMM.

Ressalte-se que os resíduos não apresentaram distribuição normal, tal condição não crítica e pôde ser relaxada, dado o tamanho da amostra, conforme Greene (2012), considerando-se propriedade de estimadores com distribuição assintótica normal, à medida que o tamanho da amostra aumenta.

\section{Resultados}

Na Tabela, 2 apresenta-se a descrição das variáveis do estudo, em que se constata que, em média, firmas da amostra exibem accruals e fluxos de caixa futuros e atuais positivos, ressaltando-se a elevada variabilidade desses atributos, conforme se observa a relação entre seus desvios padrões e suas médias. Também se constata que anos eleitorais representam cerca de $21 \%$ do período amostral considerado, respectivamente.

Tabela 2

\section{Descrição das variáveis do estudo}

\begin{tabular}{cccccc}
\hline Variável & Média & Mediana & Desvio-padrão & Mínimo & Máximo \\
\hline$F C_{i, t+1}$ & 0,061 & 0,066 & 0,111 & $-0,203$ & 0,265 \\
\hline$F C_{i, t}$ & 0,060 & 0,065 & 0,113 & $-0,204$ & 0,267 \\
\hline$A C C_{i, t}$ & 0,010 & 0,002 & 0,081 & $-0,145$ & 0,207 \\
\hline$E L E$ & 0,209 & 0 & 0,407 & 0 & 1 \\
\hline$T A M$ & 21,905 & 21,890 & 2,061 & 18,160 & 0,673 \\
\hline CRESC & 0,130 & 0,104 & 0,216 & 0,238 & 1 \\
\hline IFRS & 0,531 & 1 & 0,499 & 0 & 10,125 \\
\hline VPIB & 2,291 & 2,804 & 3,253 & $-10,894$ & 0.53 \\
\hline
\end{tabular}

Nota: Definição das variáveis: $F C_{i, t+1, k}$ é o fluxo de caixa operacional do período $t+1$ para a firma $i$ e no país $k$, escalado pelo ativo total de $t-1$ da firma $i ; F C_{i, t, k}$ é o fluxo de caixa operacional do período $t$ para a firma $i$ e no país $k$, escalado pelo ativo total de $t-1$ da firma $i ; A C C_{i, t, k}$ são os accruals no período t para a firma $i$ e no país $k ; E L E_{t, k}$ é variável dummy representativa de ano de eleições nacionais no país $k$, assumindo 1 para esses anos, e 0 em caso contrário; TAM representa o tamanho da firma, mensurada pelo log natural do ativo total; CRESC representa o crescimento da firma, mensurada pela variação percentual da receita líquida entre os períodos $t$ e t-1; IFRS é dummy representativa da adoção do padrão IFRS em cada país da amostra; VPIB corresponde à variação anual do Produto Interno Bruto (PIB). N = 4.127 observações-ano.

Fonte: dados da pesquisa. 
A Tabela 2 aponta, ainda, firmas exibindo crescimento de 13\% de suas receitas líquidas, ressaltandose o alto padrão de variabilidade dessa variável. Ademais, também se destaca que cerca de $52 \%$ do período amostral compreende período de aplicação do padrão IFRS, quando da divulgação das informações contábeis das firmas nos países da amostra, e que esses exibem, em média, variação positiva do PIB, destacando-se seu padrão de alta variabilidade. Por fim, também se destaca a variável TAM como aquela de maior homogeneidade dentre todas as variáveis quantitativas da Tabela 2.

$\mathrm{Na}$ Tabela 3, se exibem coeficientes de correlação das variáveis objeto do estudo, segregando-se os testes segundo a medida representativa da incerteza política (ELE). Constatam-se coeficientes Pearson de correlação significantes ao nível de $1 \%$ em ambos os testes executados, apontando para associação positiva entre fluxos de caixa futuros e os componentes do lucro divulgado (fluxos de caixa e accruals atuais). De outra parte, observa-se que a magnitude dos coeficientes de correlação entre as referidas variáveis aumenta ao se considerar anos de eleições nacionais, com maior efeito aparentemente registrado para o componente accruals, fornecendo indícios que parecem sinalizar efeito benéfico de períodos de maior incerteza política na capacidade preditiva de ambos os componentes do lucro.

Tabela 3

Correlações segundo período de eleições nacionais

\begin{tabular}{|c|c|c|c|c|c|c|}
\hline & \multicolumn{3}{|c|}{$E L E=0$} & \multicolumn{3}{|c|}{$E L E=1$} \\
\hline & $F C_{i, t+1}$ & $F C_{i, t}$ & $A C C_{i, t}$ & $F C_{i, t+1}$ & $F C_{i, t}$ & $A C C_{i, t}$ \\
\hline$F C_{i, t+1}$ & 1 & & & 1 & & \\
\hline$F C_{i, t}$ & $0,325^{* * *}$ & 1 & & $0,348^{* * *}$ & 1 & \\
\hline$A C C_{i, t}$ & $0,054 * * *$ & $-0,562 * * *$ & 1 & $0,111 * * *$ & $-0,567 * \star \star$ & 1 \\
\hline
\end{tabular}

Nota: Definição das variáveis: $F C_{i, t+1, k}$ é o fluxo de caixa operacional do período $t+1$ para a firma $i$ e no país $k$, escalado pelo ativo total de $t-1$ da firma $i ; F C_{i, t, k}$ é o fluxo de caixa operacional do período $t$ para a firma $i$ e no país $k$, escalado pelo ativo total de $t-1$ da firma $i ; A C C_{i t, k}$ são os accruals no período $t$ para a firma $i$ e no país $k$.

*** Significância ao nível de $1 \%$.

Fonte: dados da pesquisa.

Tomados em conjunto, esses achados fornecem indícios de que a associação entre futuros fluxos de caixa e os componentes dos lucros divulgados se altera segundo o evento incerteza política, contudo a direção do efeito parece ser de incremento da capacidade preditiva daqueles componentes em períodos de incerteza política.

Aprofunda-se a investigação pelo exposto na Tabela 4, apresentando-se os resultados para testes da influência da incerteza política na capacidade preditiva dos componentes fluxo de caixa e accruals atuais. $\mathrm{Na}$ estimação da regressão, as variáveis fluxo de caixa operacional atual $\left(F C_{i, t}\right)$, accruals $\left(A C C_{i, t}\right)$, tamanho (TAM) e crescimento (CRESC) da firma foram identificadas como endógenas, utilizando-se, então, a partir da segunda defasagem, essas variáveis como instrumentos. A variável representativa do período de incerteza política (ELE) e de interação desses períodos com as variáveis fluxos de caixas atuais e accruals foram tratadas como exógenas, assim como as variáveis representativas da variação do PIB (VPIB), do padrão IFRS (IFRS) e as dummies de anos (D_ANO), setores (D_SETOR) e países (D_PAÍSES). 
Tabela 4

Capacidade preditiva dos componentes do lucro e incerteza política

\begin{tabular}{|c|c|c|c|}
\hline \multicolumn{4}{|c|}{ Variável dependente: $\mathrm{FC}_{\mathrm{t}+1}$} \\
\hline Variáveis & Coeficientes & Erro Padrão & P-value \\
\hline$F C_{t}$ & 0,783 & 0,090 & 0,000 \\
\hline$A C C_{t}$ & 0,787 & 0,236 & 0,001 \\
\hline$E L E_{t}$ & 0,017 & 0,009 & 0,064 \\
\hline$E L E_{t} * F C_{t}$ & $-0,199$ & 0,103 & 0,053 \\
\hline$E L E_{t} * A C C_{t}$ & $-0,140$ & 0,232 & 0,547 \\
\hline TAM & 0,013 & 0,007 & 0,065 \\
\hline CRESC & $-0,085$ & 0,043 & 0,050 \\
\hline IFRS & 0,010 & 0,009 & 0,271 \\
\hline$V P I B$ & $-0,001$ & 0,001 & 0,521 \\
\hline Intercepto & $-0,332$ & 0,141 & 0,018 \\
\hline Dummies de anos & & Sim & \\
\hline Dummies de setores & & Sim & \\
\hline Dummies de países & & Sim & \\
\hline Observações-ano & & 4.127 & \\
\hline Número de instrumentos & & 108 & \\
\hline Número de firmas & & 386 & \\
\hline Estat. Wald & & $3.599,48 * * *$ & \\
\hline $\mathrm{AR}(1)$ & & $-5,69 * * *$ & \\
\hline $\mathrm{AR}(2)$ & & 1,11 & \\
\hline Hansen test & & 74,31 & \\
\hline
\end{tabular}

Notas: Modelo: $F C_{i, t+1, k}=\beta_{0}+\beta_{1} F C_{i, t, k}+\beta_{2} A C C_{i, t, k}+\beta_{3} E L E_{t, k}+\beta_{4} F C_{i, t, k} * E L E_{t, k}+\beta_{5} A C C_{i, t, k} * E L E_{t, k}+\sum_{n=6}^{N} \beta_{n} C O N T R O L E S+\varepsilon_{i t}$. Definição das variáveis: $F C_{i, t+1, k}$ é o fluxo de caixa operacional do período $t+1$ para a firma $i$ e no país $k$, escalado pelo ativo total de $t-1$ da firma $i ; F C_{i, t, k}$ é o fluxo de caixa operacional do período $t$ para a firma $i$ e no país $k$, escalado pelo ativo total de $t-1$ da firma $i ; A C C_{i, t, k}$ são os accruals no período $t$ para a firma $i$ e no país $k ; E L E_{t, k}$ é variável dummy representativa de ano de eleições nacionais no país $k$, assumindo 1 para esses anos, e 0 em caso contrário; $E L E_{t, k} * F C_{i, t, k}$ representa interação para $F C_{i, t, k}$ em anos de eleições nacionais; $E L E_{t, k} * A C C_{i, t, k}$ representa interação para $A C C_{i, t, k}$ em anos de eleições nacionais; CONTROLES se refere a matriz de variáveis controles utilizadas, quais sejam, o log natural do ativo total (TAM), a variação percentual da receita líquida entre os períodos $t$ e $t-1$ (CRESC), dummy representativa do período IFRS, a variação anual do Produto Interno Bruto (PIB) e dummies para países, anos e setores considerados na amostra.

*** Significância ao nível de $1 \%$.

Fonte: dados da pesquisa.

Pela Tabela 4, confirmam-se a capacidade de ambos os componentes do lucro - $F C_{i, t}$ e $A C C_{i, t}$ - em predizer futuros fluxos de caixa operacionais, conforme se observa os coeficientes positivos e significantes a $0,1 \%$ dessas variáveis, conforme já relatado em outras investigações (Barth et al., 2001; Boina \& Macedo, 2018; Lev et al., 2010). 
Em relação ao impacto da incerteza política na capacidade preditiva dos componentes do lucro divulgado (fluxos de caixas atuais e accruals), constata-se redução da capacidade preditiva do componente fluxo de caixa atual $\left(F C_{i, t}\right)$, conforme se observa o coeficiente negativo e significante (ao nível de 6\%) da variável $F C^{\star} E L E$. Ressalte-se que, na presença de períodos eleições nacionais, a capacidade preditiva do componente $F C_{i, t}$ é reduzida em cerca de $25 \%$ [(FC-ELE* $\left.\left.F C\right) / F C=(0,783-0,199) / 0,783\right]$, quando comparada à capacidade daquele componente em períodos de não eleições, o que indica um impacto não trivial de períodos de incerteza política na força preditiva do componente fluxo de caixa atual.

Tal achado confirma, portanto, o efeito adverso que se esperava, qual seja, de que períodos de maior incerteza política afetam negativamente a capacidade preditiva do componente fluxo de caixa, conforme já reportado por Leal et al. (2017) quanto aos efeitos de eventos que incrementam a incerteza associada a futuros fluxos de caixa.

De outra parte, a variável $A C C^{\star} E L E$ não se mostrou significante, embora tenha apresentado coeficiente negativo, o que não permite inferir sobre o efeito adverso da incerteza política na capacidade preditiva do componente accruals. Esperava-se, contudo, menor capacidade preditiva do componente accruals em torno do evento incerteza política, o que se alinharia às evidências apresentadas por Leal et al. (2017) no contexto de evento associado ao incremento de incerteza.

Destaque-se, ainda, que as variáveis TAM e CRESC mostraram-se significantes em impactar futuros fluxos de caixa, conforme já sinalizado em pesquisas anteriores (Choi et al., 2015; Farshadfar et al., 2008). Por outro lado, IFRS e VPIB não se mostraram significantes em afetar futuros fluxos de caixa, conforme se observa seus coeficientes não significantes na Tabela 4 .

De forma a se examinar a robustez dos achados, testou-se se os resultados da Tabela 4 são sensíveis à proxy utilizada para incerteza política. Para tanto, estimou-se o modelo (1) com configurações similares às reportadas na Tabela 4 , substituindo-se a medida ELE e suas interações com os componentes do lucro divulgado, adicionando-se medida representativa do índice de incerteza política econômica $(E P U)$ de cada país da amostra (com exceção da Argentina, que não possui dados para esta medida), considerando, ainda, sua interação com aqueles componentes, tal medida foi desenvolvida por Baker et al. (2016) e está disponível em Economic Policy Uncertainty (2020). Os resultados apontaram para efeito negativo da incerteza política econômica $(E P U)$ na capacidade preditiva de ambos os componentes do lucro, sugerindo que os achados parecem sensíveis à medida utilizada para a incerteza política quando se considera o componente accruals. Embora essa evidência seja relevante, vale ressaltar que a aplicação da medida $E P U$ a apenas 3 países da amostra limita a comparação efetiva com os resultados da Tabela 4 .

Assim, de forma adicional, testou-se se o impacto da incerteza política na capacidade dos componentes fluxo de caixa atual e accruals em predizer futuros fluxos de caixa também se daria em mais de um período à frente. Para tanto, estimou-se o modelo (1) com configurações similares às reportadas na Tabela 4, considerando-se como variável dependente o fluxo de caixa de dois períodos à frente $(t+2)$. Os resultados apontaram, novamente, para efeito negativo da incerteza política (ELE) na capacidade preditiva de ambos os componentes do lucro.

Tomados em conjunto, os testes de robustez confirmam o efeito negativo da incerteza política na capacidade preditiva do componente fluxo de caixa atual, conforme já reportado na Tabela 4 , bem como fornecem indícios de efeito adverso daquele evento na capacidade preditiva do componente accruals.

Com base nos achados, não se rejeita a hipótese de que a incerteza política afeta negativamente a capacidade dos componentes do lucro divulgado - accruals e fluxo de caixa - em predizer futuros fluxos de caixa, tal efeito, contudo, apenas captado conclusivamente quanto ao componente fluxo de caixa atual. Podese inferir, portanto, que a capacidade do componente fluxo de caixa atual em predizer futuros fluxos de caixa se associa negativamente a períodos de maior incerteza política, refletidos em períodos de eleições nacionais. 


\section{Conclusão}

Objetivou-se investigar a interação entre períodos de maior incerteza política e a capacidade dos componentes do lucro divulgado em predizer futuros fluxos de caixa no contexto de mercados de capitais da América Latina. Essa interação seria esperada segundo os argumentos de que a incerteza política incrementa tanto a incerteza associada a futuros fluxos de caixa das firmas quanto a possibilidade de manipulação dos componentes dos lucros por parte de gestores, o que contribuiria para redução da capacidade de ambos os componentes do lucro divulgado - fluxos de caixa e accruals atuais - em predizer futuros fluxos de caixa.

Considerou-se períodos de eleições nacionais - ano de ocorrências das eleições nacionais - como proxy para representar períodos de maior incerteza política, ao passo que a equação de previsão de fluxos de caixa de um período à frente foi utilizada para captar a capacidade preditiva dos componentes do lucro segundo os períodos de incerteza política.

As evidências coletadas sugerem que períodos de incerteza política diferenciam a capacidade dos componentes do lucro em predizer futuros fluxos de caixa, especificamente contribuem para menor capacidade preditiva do componente fluxo de caixa atual durante o período de eleições nacionais. Essas evidências se mostraram robustas, tanto a medida alternativa de incerteza política quanto a configuração alternativa do período de previsão de futuros fluxos de caixa.

Por outro lado, o componente accruals não se mostrou significante em afetar negativamente a predição de futuros fluxos de caixa em períodos de eleições nacionais, não se alinhando a noção de que o incremento de incerteza se associaria a menor capacidade desse componente em predizer futuros fluxos de caixa. Contudo, tal evidência parece sensível à proxy utilizada ao evento incerteza política e ao período de previsão definido na equação de previsão de futuros fluxos de caixa, uma vez que se constatou redução da força preditiva daquele componente em ambas as configurações de testes.

Com base nas evidências, pode-se concluir que a incerteza política afeta a capacidade preditiva dos componentes do lucro divulgado, contudo a direção desse efeito se mostra diferenciada segundo o componente e segundo a medida adotada como representante da incerteza política. A despeito desse fato, pode-se concluir que o componente fluxo de caixa atual é afetado negativamente por períodos em torno de eleições nacionais.

Avança-se na literatura internacional por fornecer evidências que incrementam a literatura relacionada à hipótese de incerteza política, especificamente quanto a seus efeitos adversos na utilidade das informações disponibilizadas pelas firmas no contexto de mercados emergentes. A esse respeito, o estudo complementa os achados acerca do impacto negativo da incerteza política na qualidade das informações contábeis divulgadas já relatados em Chen, Chen et al. (2018) e Yung e Root (2019).

Destaque-se que esse impacto negativo mostrou-se persistente, mesmo ao se considerar países específicos da América Latina, os quais exibem características distintas de outros países considerados nas pesquisas anteriores. Nessa linha, os achados relatados confirmam a relevância do evento incerteza política em diferentes mercados de capitais, caracterizando, portanto, seu caráter pervasivo. Considerando-se, ainda, o evento incerteza política em diferentes mercados, há argumentação em torno de maior impacto desse evento em mercados emergentes, quando comparados a mercados desenvolvidos (Diamonte, Liew \& Steven, 1998), contudo não se endereçou testes explícitos nessa direção e, portanto, sugere-se como futura agenda de pesquisa.

Ademais, também se complementa as evidências relatadas em Leal et al. (2017) para o contexto de mercados emergentes, fornecendo-se evidências que permitam maior esclarecimento do impacto de eventos que incrementam a incerteza associada a futuros fluxos de caixa na disponibilidade de informações úteis aos agentes dos mercados de capitais. 
Convém destacar que as evidências fornecem implicações práticas diretas para agentes dos mercados de capitais da América Latina, especificamente no que concerne ao impacto adverso da incerteza política na predição de futuros fluxos de caixa. Nesse aspecto, as evidências apontam para a necessidade de se considerar anos de eleições como atributo relevante em reduzir a capacidade preditiva do componente fluxo de caixa atual quando da predição de futuros fluxos de caixa.

A pesquisa limitou-se basicamente a captar o efeito incerteza política apenas se considerando períodos de eleições nacionais e, de forma limitada, índice de incerteza da economia política (EPU). Outras medidas, por outro lado, como índices de risco político e de crise políticas, podem ser utilizadas como proxies alternativas para captar aquele efeito, o que se sugere para futuras pesquisas. Também representa oportunidade para futuras pesquisas, no contexto da limitação acima, examinar se eventos em torno da mudança efetiva de governo ou de outros atributos relacionados às eleições também afetariam a qualidade da informação contábil.

Ademais, não se testou o efeito interativo do impacto da incerteza política na capacidade preditiva dos componentes dos lucros considerando-se, ainda, a manipulação desses componentes por parte de gestores, o que também se sugere para futuras investigações.

É provável que, em outros mercados emergentes, com configuração similar aos países amostrados nesta pesquisa, também se capte o efeito da incerteza política na capacidade preditiva dos componentes do lucro, fato que fornece sinalização para futuras investigações.

\section{Referências}

Alexakis, P., \& Petrakis, P. (1991). Analysing stock market behaviour in a small capital market. Journal of Banking \& Finance, 15(3), 471-483. doi: https://doi.org/10.1016/0378-4266(91)90081-V

An, H., Chen, Y., Luo, D., \& Zhang, T. (2016). Political uncertainty and corporate investment: evidence from China. Journal of Corporate Finance, 36, 174-189. doi: 10.1016/j.jcorpfin.2015.11.003

Arellano, M., Bond, S. (1991). Some tests of specification for panel data: Monte Carlo evidence and an application to employment equations. The Review of Economic Studies, 58(2), 277-297. doi: https:// doi.org/10.2307/2297968

Ashton, D., \& Trinh, C. (2018). Evaluating the information content of earnings forecasts. Accounting and Business Research, 48(6), 674-699. doi: https://doi.org/10.1080/00014788.2017.1415800

Baker, S. R., Bloom, N., \& Davis, S. J. (2016). Measuring economic policy uncertainty. The quarterly journal of economics, 131(4), 1593-1636. doi: https://doi.org/10.1093/qje/qjw024

Ball, R, \& Brown, P. (1968). An empirical evaluation of accounting numbers. Journal of Accounting Research, 6(2), 159-178. doi: https://doi.org/10.2307/2490232

Banco Mundial. (2018). Recuperado de https://data.worldbank.org/indicator

Barros, L. A. B. C., Bergmann, D. R., Castro, F. H., \& Silveira, A. D. (2020). Endogeneity in panel data regressions: methodological guidance for corporate finance researchers. Revista Brasileira de Gestão de Negócios, 22(Edição Especial), 437-461. doi: https://doi.org/10.7819/rbgn.v22i0.4059

Barth, M. E., Beaver, W. H., Hand, J. R. M., \& Landsman, W. R. (1999). Accruals, cash flows, and equity values. Review of Accounting Studies, 4(3-4), 205-229. doi: https://doi.org/10.1023/A:1009630100586

Barth, M. E., Clinch, G., \& Israeli, D. (2016). What do accruals tell us about future cash flows?. Review of Accounting Studies, 21(3), 768-807. doi: https://doi.org/10.1007/s11142-016-9360-4

Barth, M. E., Cram, D. P., \& Nelson, K. K. (2001). Accruals and the Prediction of Future Cash Flows. The Accounting Review, 76(1), 27-58. 
Beaver, W. (1968). The information content of annual earnings announcements. Journal of Accounting Research, 6(Supplement), 67-92. doi: https://doi.org/10.2307/2490070

Beaver, W. H., Mcnichols, M. F., \& Wang, Z. Z. (2018). The information content of earnings announcements: new insights from intertemporal and cross-sectional behavior. Review of Accounting Studies, 23(1), 95-135. doi: https://doi.org/10.1007/s11142-017-9417-z

Boina, T. M., \& Macedo, M. A. S. (2018). Capacidade preditiva de accruals antes e após as IFRS no mercado acionário brasileiro. Revista Contabilidade \& Finanças, 29(78), 375-389. doi: https://doi. org/10.1590/1808-057x201806300

Boutchkova, M., Doshi, H., Durnev, A., \& Molchanov, A. (2012). Precarious politics and return volatility. The Review of Financial Studies, 25(4), 1111-1154. doi: https://doi.org/10.1093/rfs/hhr100

Brogaard, J., \& Detzel, A. (2015). The asset-pricing implications of government economic policy uncertainty. Management Science, 61(1), 3-18. doi: http://dx.doi.org/10.1287/mnsc.2014.2044

Brown, P., Preiato, J., \& Tarca, A. (2014). Measuring country differences in enforcement of accounting standards: An audit and enforcement proxy. Journal of Business Finance \& Accounting, 41(1-2), 1-52. doi: http://dx.doi.org/10.1111/jbfa.12066

Chen, Y., Chen, D., Wang, W., \& Zheng, D. (2018). Political uncertainty and firms' information environment: Evidence from China. Journal of Accounting and Public Policy, 37(1), 39-64. doi: https://doi.org/10.1016/j.jaccpubpol.2018.01.005

Chen, F., Hope, O. K., Li, Q., \& Wang, X. (2018). Flight to quality in international markets: investors' demand for financial reporting quality during political uncertainty events. Contemporary Accounting Research, 35(1), 117-155. doi: https://doi.org/10.1111/1911-3846.12355

Choi, W., Han, S., Jung, S. H., \& Kang, T. (2015). CEO's operating ability and the association between accruals and future cash flows. Journal of Business Finance \& Accounting, 42(5-6), 619-634. doi: https://doi.org/10.1111/jbfa.12118

Dai, L., \& Ngo, P. (2020). Political uncertainty and accounting conservatism. European Accounting Review, 30(2), 277-307. doi: https://doi.org/10.1080/09638180.2020.1760117

Dechow, P. M., \& Dichev, I. D. (2002). The quality of accruals and earnings: The role of accrual estimation errors. The Accounting Review, 77(s-1), 35-59. doi: https://doi.org/10.2308/accr.2002.77.s-1.35

Dechow, P., Ge, W., \& Schrand, C. (2010). Understanding earnings quality: A review of the proxies, their determinants and their consequences. Journal of accounting and economics, 50(2), 344-401. doi: https://doi.org/10.1016/j.jacceco.2010.09.001

Dechow, P. M., Hutton, A. P., Kim, J. H., \& Sloan, R. G. (2012). Detecting earnings management: A new approach. Journal of accounting research, 50(2), 275-334. doi: https://doi.org/10.1111/j.1475-679X.2012.00449.x

Dechow, P. M., Kothari, S. P., \& Watts, R. L. (1998). The relation between earnings and cash flows. Journal of accounting and Economics, 25(2), 133-168. doi: https://doi.org/10.1016/S0165-4101(98)00020-2

Dechow, P. M., Sloan, R. G., \& Sweeney, A. P. (1995). Detecting earnings management. The Accounting Review, 70(2), 193-225.

Diamonte, R. L., Liew, J. M., Stevens, R. L. (1998). Political risk in emerging and developed markets. In Emerging Market Capital Flows. Springer, Boston, MA, Recuperado de https://books.google.com. br/books?hl=pt-BR\&lr=\&id=VzbrBwAAQBAJ\&oi=fnd\&pg=PA276\&ots=LCHcis8oXR\&sig=Ujq yzrZzUoZYVIAM9CDwDhMCYIY\#v=onepage\&q\&f=false

Economic Policy Uncertainty. Recuperado de http://www.policyuncertainty.com/ 
Farshadfar, S., Ng, C., \& Brimble, M. (2008). The relative ability of earnings and cash flow data in forecasting future cash flows. Pacific Accounting Review, 20(3), 254-268. doi: http://dx.doi. org/10.1108/01140580810920236

Fundo Monetário Internacional. Recuperado de https://www.imf.org/external/index.htm

Gao, P., \& Qui, Y. (2014). Political Uncertainty and Public Financing Costs: Evidence from U.S. Gubernatorial Elections and Municipal Bond Market. Australasian Finance and Banking Conference, Sydnei, Australia. doi: http://dx.doi.org/10.2139/ssrn.1992200

Greene, W. H. (2012). Econometric analysis (7a Ed.). Boston: Pearson Education.

Hope, O., Thomas, W. B., \& Vyas, D. (2016). Stakeholder demand for accounting quality and economic usefulness of accounting in US private firms. Journal of Accounting and Public Policy, 36(1), 1-13. doi: http://dx.doi.org/10.1016/j.jaccpubpol.2016.11.004

Jones, J. J. (1991). Earnings management during import relief investigations. Journal of Accounting Research, 29(2), 193-228. doi: https://doi.org/10.2307/2491047

Jordan, C. E., Waldron, M. A., \& Clark, S. J. (2007). An analysis of the comparative predictive abilities of operating cash flows, earnings, and sales. Journal of Applied Business Research (JABR), 23(3). doi: https://doi.org/10.19030/jabr.v23i3.1390

Julio, B., \& Yook, Y. (2012). Political uncertainty and corporate investment cycles. The Journal of Finance, 67(1), 45-83. doi: https://doi.org/10.1111/j.1540-6261.2011.01707.x

Kim, M., \& Kross, W. (2005). The ability of earnings to predict future operating cash flows has been increasing-not decreasing. Journal of Accounting research, 43(5), 753-780. doi: https://doi. org/10.1111/j.1475-679X.2005.00189.x

La Porta, R., Lopez-de-Silanes, F., Shleifer, A., \& Vishny, R. W. (1998). Law and finance. The Journal of Political Economy, 106(6), 1113-1155. doi: http://dx.doi.org/10.1086/250042

Leal, L. T. Y., Girão, L. F. D. A. P., Lucena, W. G. L., \& Martins, V. G. (2017). Persistence, value relevance, and accruals quality in extreme earnings and cash flow situations. Revista de Administração Mackenzie, 18(3), 203-231. doi: http://dx.doi.org/10.1590/1678-69712017/administracao.v18n3p203-231

Leuz, C., Nanda, D., \& Wysocki, P. D. (2003). Earnings management and investor protection: an international comparison. Journal of financial economics, 69(3), 505-527. doi: https://doi. org/10.1016/S0304-405X(03)00121-1

Lev, B. (1989). On the usefulness of earnings and earnings research: Lessons and directions from two decades of empirical research. Journal of accounting research, 27, 153-192. doi: https://doi.org/10.2307/2491070

Lev, B., Li, S., \& Sougiannis, T. (2010). The usefulness of accounting estimates for predicting cash flows and earnings. Review of Accounting Studies, 15(4), 779-807. doi: https://doi.org/10.1007/s11142-009-9107-6

Machado, M. A. V., Filho, Silva, A. C., \& Callado, A. L. C. (2014). O processo de convergência às IFRS e a capacidade do lucro e do fluxo de caixa em predizer os fluxos de caixa futuro: evidências no mercado brasileiro. Revista de Contabilidade e Organizações, 8(21), 4-13. doi: https://doi.org/10.11606/rco. v8i21.55603

Mcnichols, M. F. (2002). Discussion of the Quality of Accruals and Earnings: The Role of Accrual Estimation Errors. The Accounting Review, 77(Supplement), 61-69.

Minton, B. A., Schrand, C. M., \& Walther, B. R. (2002). The role of volatility in forecasting. Review of Accounting Studies, 7(2-3), 195-215. doi: https://doi.org/10.1023/A:1020226118973

Monfared, S., \& Pavlov, A. (2019). Political Risk Affects Real Estate Markets. The Journal of Real Estate Finance and Economics, 58(1), 1-20. doi: https://doi.org/10.1007/s11146-017-9619-y 
Moreira, J. C. C. (2018). Efeitos dos aspectos institucionais na anomalia dos accruals, Tese de doutorado, Faculdade de Economia, Administração e Contabilidade, Universidade de São Paulo.Moura, A. A. F., Altuwaijri, A., \& Gupta, J. (2020). Did mandatory IFRS adoption affect the cost of capital in Latin American countries?. Journal of International Accounting, Auditing and Taxation, 38, 100301. doi: https://doi.org/10.1016/j. intaccaudtax.2020.100301

Passos, L., \& Coelho, A. (2019). Conservadorismo condicional em função de demanda e sinalização informacional no mercado brasileiro. Revista Universo Contábil, 15(1), 131-150. doi: https://doi. org/10.4270/ruc.2019107

Pastor, L., \& Veronesi, P. (2012). Uncertainty about government policy and stock prices. The Journal of Finance, 67(4), 1219-1264. doi: https://doi.org/10.1111/j.1540-6261.2012.01746.x

Paulo, E., Martins, E., \& Pontes Girão, L. F. D. A. (2014). Accounting information quality in Latin-and North-American public firms. In Accounting in Latin America (pp. 1-39). Emerald Group Publishing Limited. doi: https://doi.org/10.1108/S1479-356320140000014001

Pincus, M., Rajgopal, S., \& Venkatachalam, M. (2007). The accrual anomaly: International evidence. The Accounting Review, 82(1), 169-203. doi: https://doi.org/10.2308/accr.2007.82.1.169

Roychowdhury, S. (2006). Earnings management through real activities manipulation. Journal of accounting and economics, 42(3), 335-370. doi: https://doi.org/10.1016/j.jacceco.2006.01.002

Sloan, R. (1996). Do stock prices fully reflect information in accruals and cash flows about future earnings. The Accounting Review, 71(3), 289-315.

Windmeijer, F. (2005). A finite sample correction for the variance of linear efficient two-step GMM estimators. Journal of econometrics, 126(1), 25-51. doi: https://doi.org/10.1016/j.jeconom.2004.02.005

Wolk, H. I., Dodd, J. L., \& Tearney, M. J. (2004). Accounting Theory: Conceptual Issues in a Political and Economic Environment (6th ed.). Thomson.

World Federation of Exchange. Recuperado de https://www.world-exchanges.org/

Yung, K., \& Root, A. (2019). Policy uncertainty and earnings management: International evidence. Journal of Business Research, 100, 255-267. doi: https://doi.org/10.1016/j.jbusres.2019.03.058 